\title{
Sub-200-kHz single soliton generation in a long ring Er-fiber laser with strict polarization control by using twisted fiber
}

\author{
L.A. Rodríguez-Morales ${ }^{\mathrm{a}}$, B. Ibarra-Escamilla ${ }^{\mathrm{a}}$, I. Armas-Riveraa ${ }^{\mathrm{a}, *}$, O. Pottiez ${ }^{\mathrm{b}}$, M.V. Andrés ${ }^{\mathrm{d}}$, \\ M. Durán-Sánchez ${ }^{\mathrm{a}, \mathrm{c}}$, E.A. Kuzin ${ }^{\mathrm{a}}$ \\ ${ }^{a}$ Instituto Nacional de Astrofísica, Óptica y Electrónica (INAOE), L. E. Erro 1, Sta. Ma. Tonantzintla, Pue. 72824, Mexico \\ ${ }^{\mathrm{b}}$ Centro de Investigaciones en Óptica (CIO), Loma del Bosque 115, Col. Lomas del Campestre, León, Gto. 37150, Mexico \\ CONACYT-Instituto Nacional de Astrofísica, Óptica y Electrónica, A. P. 51 y 216, CP 72000, Puebla, Pue., Mexico \\ ${ }^{d}$ Departamento de Física Aplicada y Electromagnetismo, ICMUV, Universidad de Valencia, C/Dr. Moliner, 50, 46100 Burjassot, Spain
}

\begin{abstract}
A B S T R A C T
In the present work we demonstrate a novel single-soliton ultra-low pulse repetition frequency passively modelocked erbium-doped fiber laser. We mitigate the residual linear birefringence of fiber by fiber twist to achieve a strict control of polarization. For mode-locking the nonlinear polarization rotation (NPR) was used. Special technique was applied to reduce the overdriving of NPR that allows the generation of single soliton in ultra-long cavity. The strict control of polarization yields a stable relation between the polarization state of the pulses propagating in the cavity and the regimes of generation. A 192.12-kHz train of soliton pulses was obtained with pulse duration of $4.7 \mathrm{ps}$ at $1560.1 \mathrm{~nm}$, the average power was $29 \mu \mathrm{W}$ and the estimated peak power was $\sim 30.8 \mathrm{~W}$ with an energy of $150.9 \mathrm{pJ}$.
\end{abstract}

\section{Introduction}

Pulsed fiber lasers are attractive for many applications such as optical communications, biological investigations, metrology, light detection and ranging (LIDAR), and machinery [1]. Some important applications, for example in biophotonics [2], require pulses with low pulse repetition frequency and high peak power. Low repetition rate helps to reduce heat accumulation $[3,4]$ that can result in damage of the material. The pulse repetition frequency can be reduced by using pulse pickers such as Pockels cells or acousto-optic modulators. Nevertheless, energetically these methods are inefficient and increase the complexity of the cavity. The fundamental pulse repetition frequency of a passively mode-locked fiber ring laser is given by $f=c / n L$, where $c$ is the speed of light in vacuum, $n$ is the fiber refractive index and $L$ is the total cavity length. In this way, it is possible to reduce the pulse repetition frequency simply by increasing the fiber length in the cavity.

Until now most attention has been given to long cavity $\mathrm{Yb}$ fiber lasers [5-11] which operate at normal dispersion and are capable to generate high-energy pulses. The first studies of long cavity lasers [5-7] demonstrated the generation of pulses with energy as high as $4 \mu \mathrm{J}$ with pulse repetition frequency as low as $37 \mathrm{kHz}$ [7], however the pulse duration was in the ns range. Later it was demonstrated that long-cavity normal dispersion lasers are capable to generate high-energy pulses with giant linear chirp [8], which can be compressed to obtain highenergy sub-picosecond pulses, from $150 \mathrm{ps}$ to $670 \mathrm{fs}$ [8] and from $3.8 \mathrm{ps}$ to $93 \mathrm{fs}$ [9]. A long-cavity Er-doped fiber laser with normal net dispersion was also reported [12]. In this work $1.7-\mu \mathrm{J}$ pulses at $35.1 \mathrm{kHz}$ pulse repetition frequency were generated.

Although most research efforts focus on long-cavity lasers with 
normal net cavity dispersion, fiber soliton sources operating in the anomalous dispersion regime are attractive as well because of their capacity of generating ultrashort pulses in the wavelength range from 1530 to $2000 \mathrm{~nm}$ using Er-, Tm-, Ho-doped fibers and the possibility to tune the wavelength using the effect of soliton self-frequency shift. However, only few works can be found where the generation of solitons with low pulse repetition frequency was demonstrated [13-15]. In [13], solitons with pulse duration of $0.919 \mathrm{ps}$ and pulse repetition frequency of $0.961 \mathrm{MHz}$ were generated by a ring cavity laser using NPR for mode-locking. A large effective area fiber (LEAF) was used in the cavity, which kept both the dispersion and nonlinear effects simultaneously low. In [14], using a ring cavity configuration, solitons with an ultralow repetition rate of $943-154 \mathrm{kHz}$ for a fiber length of $0.1-1.3 \mathrm{~km}$ were obtained. The generation of solitons with low pulse repetition frequency is the challenging task. Generally, increasing the cavity length makes the generation of a single in cavity soliton unstable. Long fiber lasers tend to generate multiple solitons in the cavity, see for example [16] where the formation of a large number of solitons in a 720-m long cavity was demonstrated. Because multiple solitons were present in the cavity, the pulse repetition frequency was high, at least higher than $10 \mathrm{MHz}$ (no precise data were provided in the paper). Long cavity lasers tend also to generate pulses with complex waveforms such as noise-like pulses (NLP) [17], rectangular-shaped pulses, etc. [18-20].

For artificial saturable absorbers based on fiber nonlinearity, such as NPR [21] and nonlinear optical/amplifying loop mirrors (NOLM/ NALMs) [17,22], where the transmission function is produced interferometrically, the slope of the transmission curve becomes negative if the pulse power or the fiber length exceed some critical value. Therefore, the generation of solitons with low repetition rate requires fibers with low nonlinearity, as in [13]. Another problem is randomly varying birefringence along the length of fibers. This uncontrolled birefringence can lead to a range of unpredictable and undesirable dynamics [23]. To achieve stability, polarization maintaining (PM) fibers can be used, as it was done in [14]. However, it is often necessary (and simpler/ more cost effective) to develop lasers using standard non-PM fiber. An alternative solution to the polarization instabilities problem caused by residual birefringence is provided by twisting the fiber. Fiber twist mitigates linear birefringence and introduces circular birefringence [24]. A pulse propagating in the twisted fiber maintains constant ellipticity of polarization, which is essential for NPR-based mode locking. Circular polarization causes a rotation of the azimuth of the polarization ellipse, however the angle of this rotation is stable and does not depend on environmental conditions. These properties of the twisted fiber allow a strict control of the polarization state in the laser cavity. Recently, we demonstrated stable and predictable switching between different regimes of generation in a mode-locked fiber ring laser where fiber twist was used to cancel residual birefringence [25,26].

Recently we proposed a new way to solve the problem of overdriving for long ring cavity lasers using NPR for mode-locking [27]. The handedness of NPR depends on the handedness of the polarization. So, if we change the handedness of the polarization after propagation through a section of the cavity, the angle of NPR acquired in this section will be compensated in the remaining part of the cavity. The laser reported in [27] includes two 200-m spans of the twisted fiber. After propagation through the first span we changed the polarization handedness using a Faraday mirror. Insertion losses between the 200-m spans ensured that the NPR compensation was not complete, allowing mode locking operation. Depending on the polarization adjustment the laser operated in different regimes including the generation of the single in cavity soliton with $467.2 \mathrm{kHz}$ repetition rate and pulse duration of $2.9 \mathrm{ps}$.

In the present work we propose and investigate a novel technique to realize the cancellation of excessive NPR in a long mode-locked fiber ring laser. We used a double-pass nonlinear segment (DPNS) in which the pulse propagates through the same fiber with the same polarization handedness, so the handedness of NPR is the same with respect to the direction of the light propagation. However in the laboratory coordinate system the handedness of NPR is opposite for forward and backward directions. The total length of the cavity is $\sim 1065 \mathrm{~m}$. In the single-soliton regime we obtained the $192.12-\mathrm{kHz}$ train of 4.7-ps pulses. The average intracavity power was $29 \mu \mathrm{W}$ with calculated peak power of $30.8 \mathrm{~W}$. To the best of our knowledge it is the lowest pulse repetition frequency the train of solitons ever reported.

\section{Experimental setup}

The experimental setup is shown in Fig. 1. It includes a double-pass amplifier (DPA) (green dashed box), a double-pass nonlinear segment (DPNS) (yellow dotted box), and elements connecting these two principal sections. The DPA includes a 45-cm long M-12 (Thorlabs) erbiumdoped fiber (EDF) pumped at $980 \mathrm{~nm}$ through a 980/1550 nm wavelength-division multiplexer (WDM), a Faraday mirror (FM), and a polarizing beam splitter (PBS).

Linearly polarized light that comes out from port 3 of the PBS goes through the in-line polarization controller PC1 (model PLC-900Thorlabs) which allows the adjustment of the polarization ellipticity through the pressure applied to the fiber. Elliptically polarized light then enters to a 50/50 coupler, where one port is used as Output 2 while the other port enters to the DPNS. The DPNS is built of a circulator C1, a 500-m long twisted SMF-28 fiber, and a circulator C2. Light entering through the port 1 of $\mathrm{C} 1$ passes to the port 2 without any ellipticity change, then it propagates through the 500-m long SMF-28

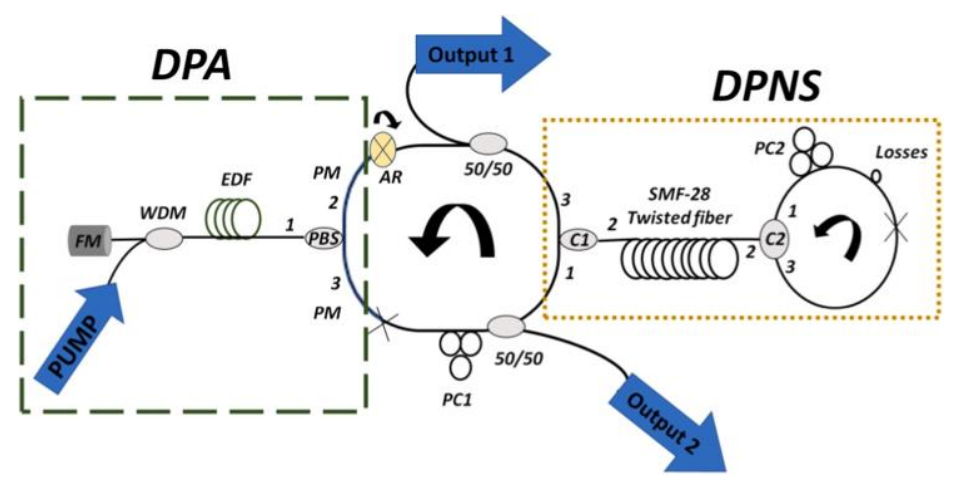

Fig. 1. Experimental setup. 
twisted fiber where NPR effect occurs. The fiber was twisted with the twist rate of 6 turns $/ \mathrm{m}$ and wounded on a $50-\mathrm{cm}$ diameter cylinder. When the fiber is twisted, the linear birefringence is canceled and the polarization ellipticity is not altered as the pulse travels through the fiber.

The angle of NPR depends on the signal ellipticity, power, and the length of the fiber $[28,29]$. The handedness of the NPR is defined by the handedness of the polarization. To counteract the effect of the NPR overdriving, we introduce at the end of the $500-\mathrm{m}$ fiber the $\mathrm{C} 2$ with spliced ports 1 and 3 . The light entering the $\mathrm{C} 2$ through the port 2 is transmitted to port 3 , then to the port 1 and finally to the port 2 without change of handedness (if the path between ports 3 and 1 has no birefringence). The handedness of NPR is the same at forward and backward propagation through SMF-28 with respect to the pulse propagation direction, however they are opposite with respect to the laboratory coordinate system. Therefore the angle of NPR acquired in the forward propagation is opposite to that acquired in the backward propagation and the total NPR angle can be equal to zero. For modelocking some NPR angle has to exist. It can be done through power asymmetry. To create power asymmetry, we introduced between ports 3 and 1 a curvature producing some loss. The total loss produced by the circulator $\mathrm{C} 2$ and the curvature is $3-\mathrm{dB}$. To compensate the birefringence caused by the curvature, the PC2 is used.

This technique different to that reported in [30] where NPR was reduced by using two different spans of the fiber through which the pulse with opposite handedness propagates. The technique presented now offers the simpler configuration and easy adjustment of NPR angle.

After double pass through The SMF-28 fiber the light from the port 2 of the $\mathrm{C} 1$ enters to the port 3 and then to the 50/50 coupler, one port of which was used as Output 1 . The other output port was spliced with the polarization maintaining port of the PBS. When incident light with any state of polarization enters to the port 2, only one linearly polarized component parallel to the PM fiber axis passes through the PBS to port 1 , so that it operates like a polarizer. Rotating the splice by an azimuth rotator (AR), the polarization orientation with respect to the axes of the PBS can be adjusted that changes the transmission through the PBS. This linearly polarized component travels through the EDF changing its polarization due to the birefringence of the fiber. Then the light is reflected by the FM, which turns the polarization of the reflected light to orthogonal to the incident light. In this way, the polarization of the reflected light is orthogonal to the polarization of the incident light at any point of the DPA, independently of the fiber birefringence. Therefore, the FM eliminates any sensitivity of the EDF birefringence to environmental conditions. The reflected light returning to port 1 of the PBS has a polarization orthogonal to that of the incident light emerging from the same port 1 , so that it is transferred to the port 3 . It is important to note that the DPA is particularly important to ensure strict polarization control in the cavity because the EDF birefringence depends on pump power [31].

When the fiber is twisted, circular birefringence is induced, a wavelength dependence appears, creating a spectral filtering effect as experimentally shown in [32]. Double-pass propagation with the same twist ratio and the same fiber length in both directions ensure that this circular birefringence effect is mitigated [30]. So both birefringence and NPR are controlled in the proposed experimental setup, which allows us to design a stable laser with reproducible results under the same initial conditions of pumping power and polarization.

The dispersion introduced by the double pass through the SMF-28 fiber within the DPNS is $18.75 \mathrm{ps} / \mathrm{nm}$. The cavity also includes $2 \mathrm{~m}$ of the OFS-980 fiber (the WDM ports), which contributes with dispersion of $-0.013 \mathrm{ps} / \mathrm{nm}$ for the double pass in the amplifier section, $0.45-\mathrm{m}$ of the EDF with dispersion of $0.006 \mathrm{ps} / \mathrm{nm}$ for the double pass, and $1.5-\mathrm{m}$ $\mathrm{PM}$ panda fiber at the polarizer input with dispersion of approximately
$0.003 \mathrm{ps} / \mathrm{nm}$. The total cavity length is $1065 \mathrm{~m}$ with net anomalous dispersion of approximately $18.85 \mathrm{ps} / \mathrm{nm}$. The output 1 is further split using three additional couplers in order to produce additional output ports. The pulse duration was measured with an autocorrelator (FR103XL), the optical spectrum was measured with an optical spectrum analyzer (Anritsu MS9710A), and the output pulses were detected by a $10-\mathrm{GHz}$ photodetector with a $1.5-\mathrm{GHz}$ oscilloscope (Agilent $54845 \mathrm{~A}$ ). Output 2 was used to monitor the polarization state inside the cavity using a polarimeter (PAX5710R3-T, Thorlabs).

\section{Results and discussions}

First, we started the laser generation in continuous wave (CW) operation and rotating the AR defined the polarization azimuths corresponding to maximal and minimal output powers. These azimuths correspond to maximal and minimal low-power transmission through the PBS. In the following the minimal transmission angle is referred to as $0^{\circ}$ and the angle of maximal transmission is $100^{\circ}$. In our previously reported works used all-polarization control cavities [25-27,33] we experimentally found that the regimes of mode-locking operation depend on the polarization state in $\mathrm{CW}$ mode, before mode-locking is started. We will refer to this state as the initial polarization state. Depending on the initial polarization state, the mode-locking operation was started in different regimes. In the present work, we focused on the regime with a single soliton in the cavity. The initial polarization conditions at which single-soliton generation is obtained are shown in Fig. 2.

In any of the points shown in Fig. 2, it is possible to find singlesoliton operation. To show the type of pulses obtained at different pump powers the polarization ellipticity was fixed at $25^{\circ}$ with azimuth of $65^{\circ}$. Since the pump power hysteresis effect [34] is presented, the laser cannot be initiated at low power. To start mode-locked operation we set the pump power to the high value of $175 \mathrm{~mW}$. At this pump power chaotic, multisoliton generation is observed.

The appearance of multiple solitons within the cavity at high pumping powers is due to the fact that the energy of a soliton is limited by the area theorem. This limit is given by the width of the pulse and dispersion of the fiber through which it propagates. The average power provided by single soliton is defined by the soliton energy multiplied by pulse repetition frequency. When the pump power increases the average power generated by the laser becomes higher than the average power provided by a single soliton and the laser must generate multiple solitons or pulses with complicated waveforms. This behavior can be reversed if the pumping power is decreased, reducing the amount of

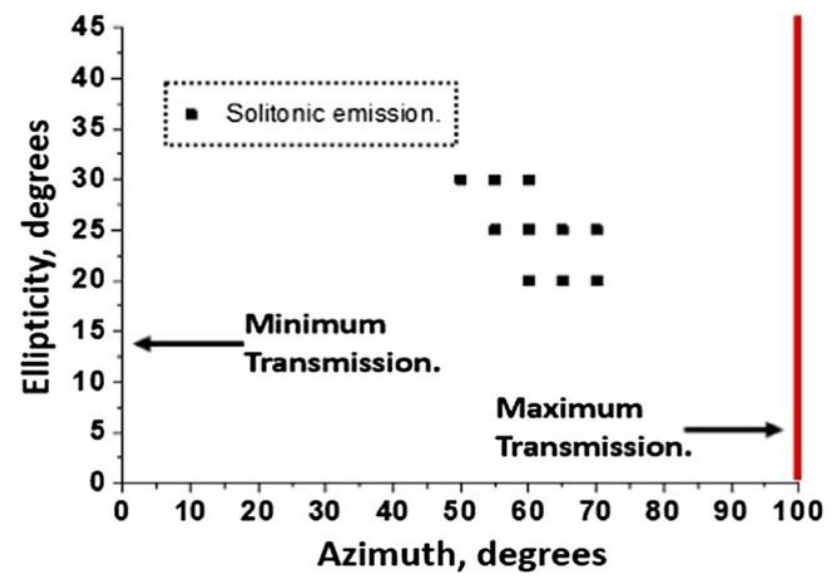

Fig. 2. Ellipticity map where solitons were found. 

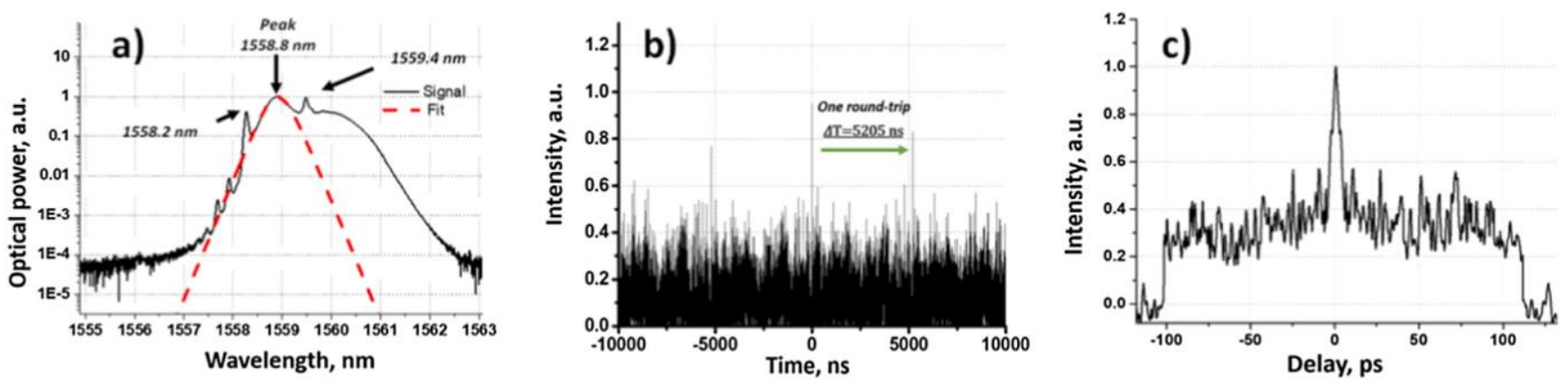

Fig. 3. Chaotic multisoliton state at $175 \mathrm{~mW}$ pump power. (a) Optical spectrum, (b) Temporal complete-randomized distribution, and (c) Autocorrelation trace.

pulses that circulate inside the cavity until a single soliton is obtained.

Fig. 3(a) shows the optical spectrum of the chaotic multisoliton operation. The spectrum shown in Fig. 3(a) is asymmetric, skewed to longer wavelengths. However, we can see that the part of the spectrum centered at $1558.8 \mathrm{~nm}$ is typical for solitons with Kelly sidebands at $1558.2 \mathrm{~nm}$ and $1559.4 \mathrm{~nm}$. The dashed line shows the fi by the spectrum of the $\mathrm{sech}^{2}$ pulse with FWHM of $0.53 \mathrm{~nm}$. The comparison between the fi curve and the measured spectrum shows some red shift of the soliton spectra, which indicates that the laser emission consists of solitons with diff durations, the shorter ones being red shifted by the soliton self-frequency shift. The mutual soliton interaction forces are very weak to cause the temporary locking between solitons. As a consequence of the weak interaction, the cavity is fi with pulses [35,36], see Fig. 3(b). The oscilloscope resolution does not allow measuring individually closely spaced pulses, however, we can see that at least the highest peaks are repeated with the periodicity corresponding to the roundtrip time of $5205 \mathrm{~ns}$. This allows the assumption that the laser generates a periodic train of pulses at least over several round trips. The autocorrelation trace shows a doublescaled structure typical for NLP. Usually the origin of the pedestal is attributed to the chaotic, nonstationary dynamics of solitons confi within the cavity [37].

At the pump power of $100 \mathrm{~mW}$ the number of pulses is reduced and pulses form bunches of solitons, see Fig. 4(b). The bunches of solitons have a duration of around 2850 ns, see inset in Fig. 4(b). Fig. 4(a) shows the optical spectrum. The spectrum is similar to that measured at pump power of $175 \mathrm{~mW}$. It has a solitonic part centered at $1559.5 \mathrm{~nm}$ (slightly redshifted in comparison with Fig. 4(a)) with Kelly sidebands at $1558.9 \mathrm{~nm}$ and $1560.15 \mathrm{~nm}$. The solitonic part of the spectrum is fitted well assuming the $\mathrm{sech}^{2}$ profile pulse with FWHM of $0.49 \mathrm{~nm}$, a value that is almost unchanged compared with the chaotic state. The autocorrelation trace is presented in Fig. 4(c). The autocorrelation traces in Figs. 3(c) and 4(c) are also similar, with a narrow central peak which rides a nearly uniform pedestal. This could again indicate that the solitons in the bunches are in random relative motion. The amplitude of the pedestal however is 2 times higher in Fig. 3(c). This difference indicates a higher density of solitons in the regime shown in Fig. 3.

At $75 \mathrm{~mW}$ of pump power, a transition to a regime of more widely spaced solitons is observed, see Fig. 5(b) which shows the waveform. As presented in Fig. 5(a), the central wavelength of the optical spectrum is $1559.9 \mathrm{~nm}$ with a FWHM of $0.45 \mathrm{~nm}$. The small red shift is still present making the spectrum slightly asymmetric. In this regime the laser tends to produce multiple pulses per round trip because pulse energy is locked to the fundamental soliton energy. When the number of pulses is less than some critical value, the solitons are separated [38]. This phenomenon causes the decrease of the pedestal of the autocorrelation trace, see Fig. 5(c).
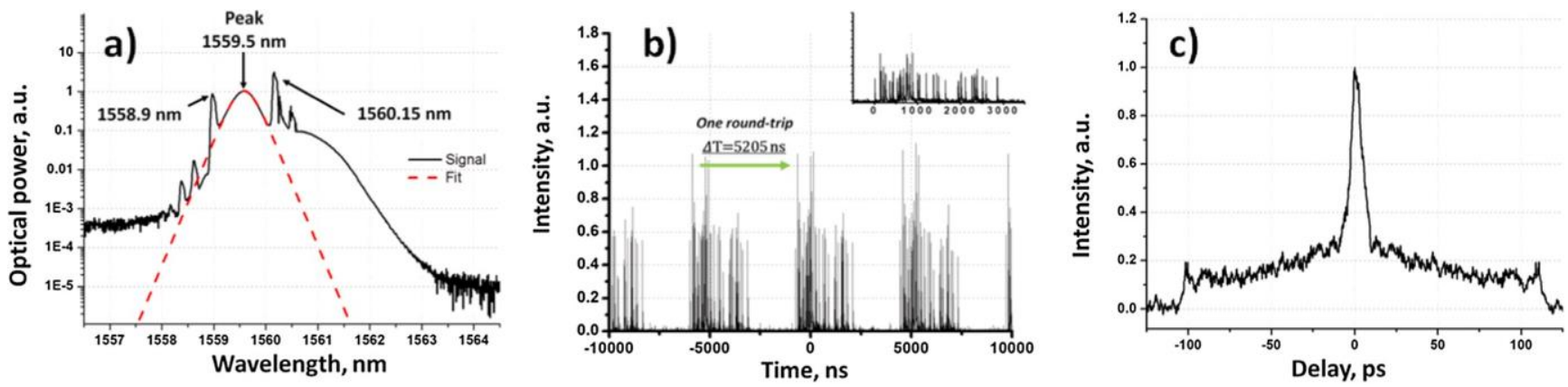

Fig. 4. Bunch solitons state at $100 \mathrm{~mW}$ pump power. (a) Optical spectrum, (b) Temporal trace, and (c) Autocorrelation trace.
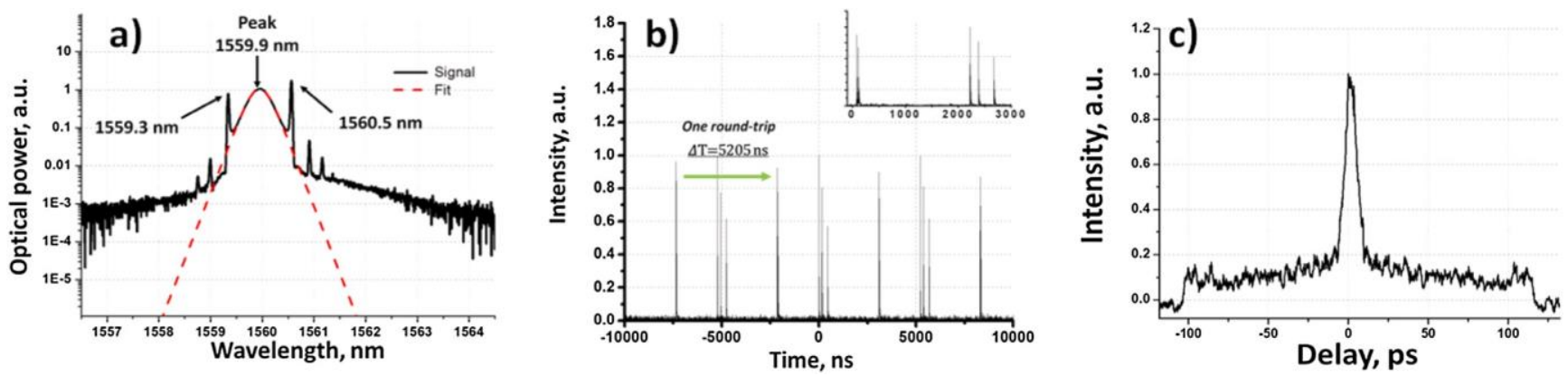

Fig. 5. Bunch of solitons at $75 \mathrm{~mW}$ of pump power. (a) Optical spectrum, (b) Temporal trace, and (c) Autocorrelation trace. 

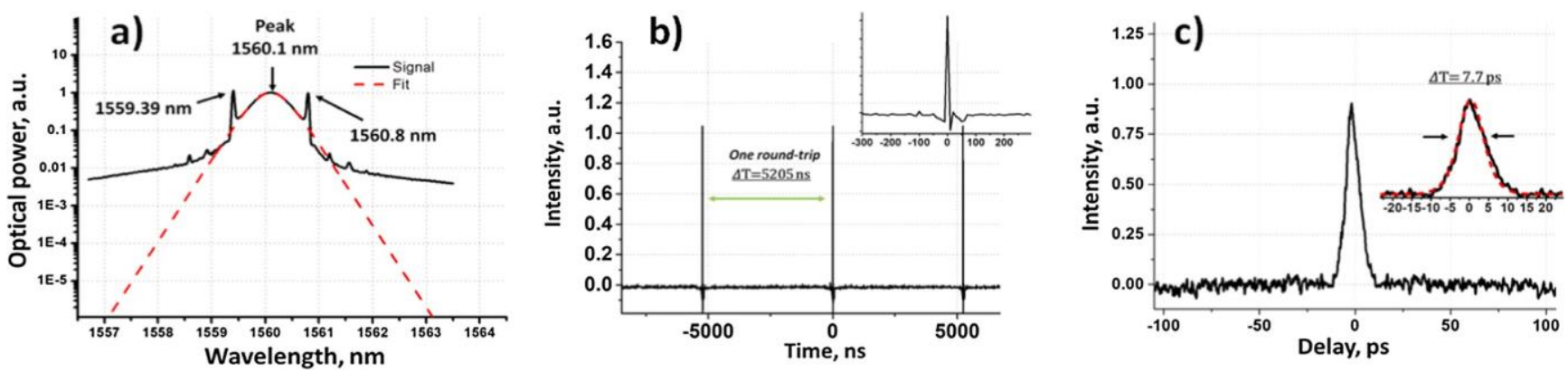

Fig. 6. Single-soliton state at $30 \mathrm{~mW}$ pump power. (a) Optical spectrum, (b) Temporal trace, and (c) Autocorrelation trace.

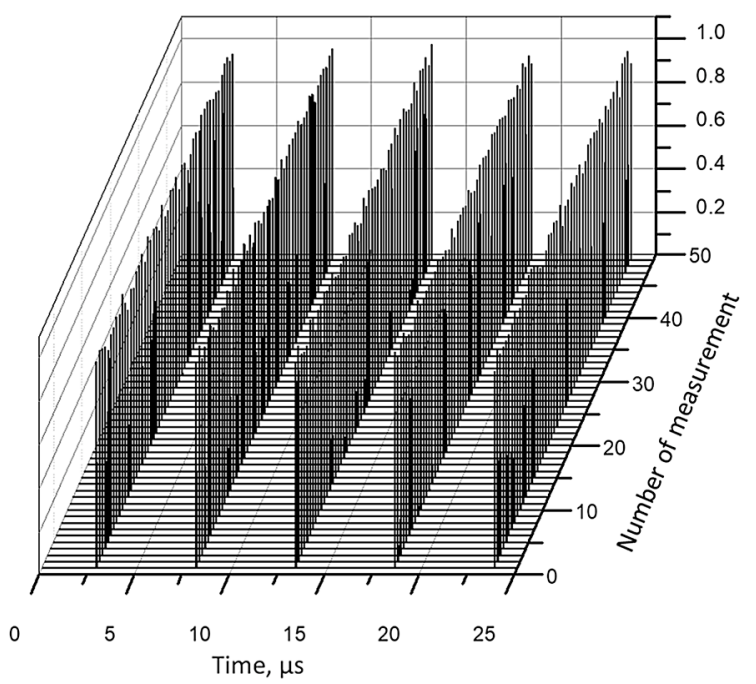

(a)

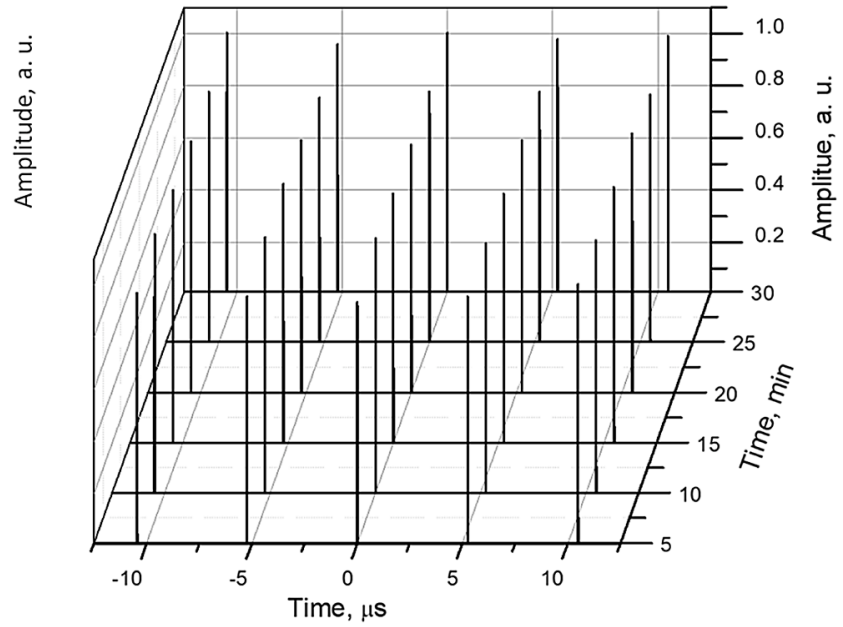

(b)

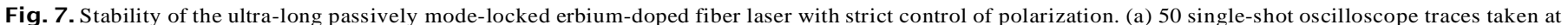
$104.73-\mu \mathrm{s}$ and (b) 6 measurements over $30 \mathrm{~min}$ with intervals of $5 \mathrm{~min}$.

The total number of pulses in the cavity gradually decreases with the output power. When the pump power is $30 \mathrm{~mW}$ the spectrum becomes typical of a soliton laser, Fig. 6(a), with the central wavelength at $1560.1 \mathrm{~nm}$, the FWHM of $0.65 \mathrm{~nm}$, and Kelly side-bands at 1559.39 and $1560.8 \mathrm{~nm}$. Fig. 6(b) shows the pulse train with a period of $5205 \mathrm{~ns}$, corresponding to a repetition rate of $192.12 \mathrm{kHz}$. The average intracavity power is $\sim 29 \mu \mathrm{W}$ and the estimated peak power is $\sim 30.8 \mathrm{~W}$ with the pulse energy of 150.9 pJ. Fig. 6(c) shows the pedestal-free autocorrelation trace, which is well fitted by the corresponding autocorrelation trace for the $\operatorname{sech}^{2}$ temporal profile.

The FWHM of the autocorrelation trace is measured to be $7.7 \mathrm{ps,}$ resulting in a pulse duration of $4.7 \mathrm{ps}$. The time-bandwidth product of 0.36 shows that the pulse is nearly transform-limited. The autocorrelation trace shows an isolated peak in the entire autocorrelation window (around $200 \mathrm{ps}$ ) without any small secondary peaks. The spectrum does not reveal any modulation which is characteristic for stable packets of solitons like soliton molecules. Therefore, in spite that there is some gap between the autocorrelator window and the oscilloscope response time, all these data give strong evidence that a single pulse is present in the cavity in this regime.

The proposed ultra-long passively mode-locked erbium-doped fiber laser with strict control of polarization presents very high stability as shown in Fig. 7. Fig. 7(a) shows 50 single-shot oscilloscope traces taken at $104.73-\mu$ intervals. Five round trips are shown. The total measurement time was $5.2 \mathrm{~ms}$. Fig. 7(b) shows 6 measurements taken every $5 \mathrm{~min}$ with total time of $30 \mathrm{~min}$. Experimentally, it has also been proven that the laser works continuously for periods larger than $6 \mathrm{~h}$.

\section{Conclusions}

In summary, we report the fiber ring laser with the decreased net nonlinear polarization rotation and the strict polarization control. The pulse type emission depends on the initial polarization conditions, the use of the twisted fiber yields the stable and reproducible single-soliton emission. In the single soliton in the cavity regime the laser generates a $4.7 \mathrm{ps}$ soliton with a repetition rate of $192.12 \mathrm{kHz}$. The intracavity average power was $29 \mu \mathrm{W}$, which gives the pulse energy of $150.9 \mathrm{pJ}$ and estimated peak power of $\sim 30.8 \mathrm{~W}$. Such ultra-low repetition rate as well as short pulse width makes this mode-locked all-fiber laser a suitable oscillator to be used as a compact source for biomedical diagnostics and micromachining because of its low average power.

\section{Funding}

Consejo Nacional de Ciencia y Tecnología, project 287315. 


\section{Acknowledgments}

I. Armas-Rivera thanks Consejo Nacional de Ciencia y Tecnología postdoctoral fellow 28667

\section{References}

[1] A. Pual, M. Chandra, Fiber Lasers: Advances in Research and Applications, Nove Science Publishers, Incorporated, 2017.

[2] K. Wang, N.G. Horton, K. Charan, C. Xu, Advanced fiber soliton sources for nonlinear deep tissue imaging in biophotonics, IEEE J. Sel. Top. Quantum Electron. 20 (2) (2014) 50-60, https://doi.org/10.1109/jstqe.2013.2276860.

[3] S.M. Eaton, H. Zhang, M.L. Ng, J. Li, W.-J. Chen, S. Ho, P.R. Herman, Transition from thermal diffusion to heat accumulation in high repetition rate femtosecond laser writing of buried optical waveguides, Opt. Express 16 (13) (2008) 9443-9458, https://doi.org/10.1364/OE.16.009443.

[4] S. Juodkazis, H. Misawa, I. Maksimov, Thermal accumulation effect in three-dimensional recording by picosecond pulses, Appl. Phys. Lett. 85 (22) (2004) 5239 5241, https://doi.org/10.1063/1.1829799.

[5] S. Kobtsev, S. Kukarin, Y. Fedotov, Ultra-low repetition rate mode-locked fiber lase with high-energy pulses, Opt. Express 16 (26) (2008) 21936-21941, https://doi. org/10.1364/OE.16.021936.

[6] X. Tian, M. Tang, X. Cheng, P.P. Shum, Y. Gong, C. Lin, High-energy wave-breakingfree pulse from all-fiber mode-locked laser system, Opt. Express 17 (9) (2009) 7222-7227, https://doi.org/10.1364/OE.17.007222.

[7] S.M. Kobtsev, S.V. Kukarin, S.V. Smirnov, Y.S. Fedotov, High-energy mode-locked all-fiber laser with ultralong resonator, Laser Phys. 20 (2) (2010) 351-356, https:// doi.org/10.1134/S1054660X10040067.

[8] W.H. Renninger, A. Chong, F.W. Wise, Giant-chirp oscillators for short-pulse fiber amplifiers, Opt. Lett. 33 (24) (2008) 3025-3027, https://doi.org/10.1364/OL.33. 003025.

[9] H. Sayinc, D. Mortag, D. Wandt, J. Neumann, D. Kracht, Sub-100 fs pulses from a low repetition rate Yb-doped fiber laser, Opt. Express 17 (7) (2009) 5731-5735, https://doi.org/10.1364/OE.17.005731.

[10] Q. Lu, J. Ma, D. Duan, X. Lin, Q. Mao, Reducing the pulse repetition rate of picosecond dissipative soliton passively mode-locked fiber laser, Opt. Express 27 (3) (2019) 2809-2816, https://doi.org/10.1364/OE.27.002809.

[11] A. Ivanenko, S. Kobtsev, S. Smirnov, A. Kemmer, Mode-locked long fibre master oscillator with intra-cavity power management and pulse energy $>12 \mu \mathrm{J}$, Opt. Express 24 (6) (2016) 6650-6655, https://doi.org/10.1364/OE.24.006650.

[12] B.N. Nyushkov, V.I. Denisov, S.M. Kobtsev, V.S. Pivtsov, N.A. Kolyada, A.V. Ivanenko, S.K. Turitsyn, Generation of $1.7-u J$ pulses at 1.55 um by a self-modelocked all-fiber laser with a kilometers-long linear-ring cavity, Laser Phys. Lett. 79 (2010) 661-665, https://doi.org/10.1002/lapl.201010037.

[13] C. Cuadrado-Laborde, J.L. Cruz, A. Díez, M.V. Andrés, Sub-picosecond ultra-low frequency passively mode-locked fiber laser, Appl. Phys. B 122 (11) (2016) 273, https://doi.org/10.1007/s00340-016-6548-Z.

[14] Y. Senoo, N. Nishizawa, Y. Sakakibara, K. Sumimura, E. Itoga, H. Kataura, K. Itoh, Ultralow-repetition-rate, high-energy, polarization-maintaining, Er-doped, ultrashort-pulse fiber laser using single-wall-carbon-nanotube saturable absorber, Opt. Express 18 (20) (2010) 20673-20680, https://doi.org/10.1364/OE.18.020673.

[15] H.Ahmad, M.F. Ismail, S.N.M.Hassan,F.D.Muhammad, M.Z.Zulkifli, S.W.Harun, Supercontinuum generation from a sub-megahertz repetition rate femtosecond pulses based on nonlinear polarization rotation technique, J. Mod. Opt. 61 (16) (2014) 1333-1338, https://doi.org/10.1080/09500340.2014.931478.

[16] X. Liu, Coexistence of strong and weak pulses in a fiber laser with largely anomalous dispersion, Opt. Express 19 (7) (2011) 5874-5887.

[17] H. Santiago-Hernandez, O. Pottiez, R. Paez-Aguirre, H.E. Ibarra-Villalon, A. Tenorio-Torres, M. Duran-Sanchez, J.C. Hernandez-Garcia, Generation and characterization of erbium-Raman noise-like pulses from a figure-eight fibre laser, Laser Phys 25 (4) (2015) 045106, https://doi.org/10.1088/1054-660X/25/4/ 045106.

[18] X. Li, X. Liu, X. Hu, L. Wang, H. Lu, Y. Wang, W. Zhao, Long-cavity passively mode- locked fiber ring laser with high-energy rectangular-shape pulses in anomalous dispersion regime, Opt. Lett. 35 (19) (2010) 3249-3251, https://doi.org/10.1364/ OL.35.003249.

[19] G. Semaan, F.B. Braham, J. Fourmont, M. Salhi, F. Bahloul, F. Sanchez, $10 \mu \mathrm{J}$ dissipative soliton resonance square pulse in a dual amplifier figure-of-eight doubleclad Er: Yb mode-locked fiber laser, Opt. Lett. 41 (20) (2016) 4767-4770, https:// doi.org/10.1364/OL.41.004767.

[20] K. Karol, G. Sobon, J. Sotor, K.M. Abramski, Fully-integrated dual-wavelength allfiber source for mode-locked square-shaped mid-IR pulse generation via DFG in PPLN, Opt. Express 23 (25) (2015) 32080-32086, https://doi.org/10.1364/OE.23 032080.

[21] V.J. Matsas, T.P. Newson, D.J. Richardson, D.J. Payne, Selfstarting passively modelocked fibre ring soliton laser exploiting nonlinear polarisation rotation, Electron Lett. 28 (15) (1992) 1391-1393, https://doi.org/10.1049/el:19920885.

[22] W.H. Cao, P.K.A. Wai, Amplification and compression of ultrashort fundamental solitons in an erbiumdoped nonlinear amplifying fiber loop mirror, Opt. Lett. 28 (4) (2003) 284-286, https://doi.org/10.1364/OL.28.000284.

[23] R I Woodward, Dispersion engineering of mode-locked fibre lasers, J. Opt. 20 (3) (2018) 033002, https://doi.org/10.1088/2040-8986/aaa9f5.

[24] R. Ulrich, A. Simon, Polarization optics of twisted single-mode fibers, Appl. Opt. 18 (13) (1979) 2241-2251, https://doi.org/10.1364/AO.18.002241.

[25] H. Santiago-Hernández, Y.E. Bracamontes-Rodríguez, G. Beltrán-Pérez, I. ArmasRivera, L.A. Rodríguez- Morales, O. Pottiez, B. Ibarra-Escamilla, M. Durán-Sánchez, M.V. Hernández-Arriaga, E.A. Kuzin, Initial conditions for dissipative solitons in a strict polarization-controlled passively mode-locked Er-Fiber laser, Opt. Express 25 (21) (2017) 25036-25045, https://doi.org/10.1364/OE.25.025036.

[26] L.A. Rodriguez-Morales, H. Santiago-Hernández, O. Pottiez, Y.E. BracamontesRodríguez, B. Ibarra-Escamilla, M. Durán-Sánchez, M.V. Hernández-Arriaga, M. Avazpour, G. Beltrán-Pérez, E.A. Kuzin, Dual-wavelength quasi-mode-locked regimes of an Er-doped fiber ring laser, OSA Continuum 1 (2) (2018) 416-425, https://doi.org/10.1364/OSAC.1.000416.

[27] L.A. Rodriguez-Morales, I. Armas-Rivera, B. Ibarra-Escamilla, O. Pottiez, H. Santiago-Hernandez, M. Durán-Sánchez, M.V. Andrés, E.A. Kuzin, Long cavity ring fiber mode-locked laser with decreased net value of nonlinear polarization rotation, Opt. Express 27 (10) (2019) 14030-14040, https://doi.org/10.1364/OE 27.014030.

[28] H. Winful, Self-induced polarization changes in birefringent optical fibers, Appl. Phys. Lett. 47 (3) (1985) 213-215, https://doi.org/10.1063/1.96221.

[29] C.R. Menyuk, Pulse propagation in an elliptically birefringent Kerr medium, IEEE J. Quantum Electron. 25 (12) (1989) 2674-2682, https://doi.org/10.1109/3.40656.

[30] C. Vinegoni, M. Wegmuller, B. Huttner, N. Gisin, Measurement of nonlinear polarization rotation in a highly birefringent optical fiber using a Faraday mirror, $\mathrm{J}$. Opt. A: Pure Appl. Opt. 2 (2000) 314-318, https://doi.org/10.1088/1464-4258/2/ $4 / 313$.

[31] Yu.O. Barmenkov, A.V. Kir'yanov, M.V. Andrés, Resonant and thermal changes of refractive index in a heavily doped erbium fiber pumped at wavelength $980 \mathrm{~nm}$, Appl. Phys. Lett. 85 (13) (2004) 2466-2468, https://doi.org/10.1063/1.1787151.

[32] L.A. Rodríguez-Morales, I. Armas-Rivera, M. Avazpour, G. Beltran-Pérez, H. Santiago-Hernandez, B. Ibarra- Escamilla, M. Durán-Sánchez, O. Pottiez, E.A. Kuzin, Experimental investigation of polarization imbalanced nonlinear loop mirror with double-sense twisted fiber as a filter to clean up solitons, J. Opt. 20 (1) (2018) 015502, https://doi.org/10.1088/2040-8986/aa9af8.

[33] Y.E. Bracamontes-Rodríguez, O. Pottiez, J.P. Lauterio-Cruz, E. García-Sanchez, J.C. Hernandez-Garcia, M. Bello-Jiménez, B. Ibarra-Escamilla, E.A. Kuzin, Polarization mapping of a dual-wavelength passively mode-locked fiber ring laser, J. Opt. 21 (4) (2019) 045504, https://doi.org/10.1088/2040-8986/ab0bf7.

[34] M. Nakazawa, E. Yoshida, Y. Kimura, Low threshold, 290 fs erbium-doped fiber laser with a nonlinear amplifying loop mirror pumped by InGaAsP laser diodes, Appl. Phys. Lett. 59 (17) (1991) 2073-2075, https://doi.org/10.1063/1.106134.

[35] F. Amrani, A. Haboucha, M. Salhi, H. Leblond, A. Komarov, F. Sanchez, Dissipative solitons compounds in a fiber laser. Analogy with the states of the matter, Appl, Phys. B 99 (1-2) (2010) 107-114, https://doi.org/10.1007/s00340-009-3774-7.

[36] K. Guesmi, G. Seeman, M. Salhi, Y. Meng, F. Bahloul, H. Leblond, F. Sanchez, Lband harmonic mode locking of single-, two-, and three-soliton bunches from Cband amplifier in a figure-of-eight fiber laser, Roman. J. Phys. 61 (7-8) (2016) 1330-1338.

[37] C. Lecaplain, P. Grelu, J.M. Soto-Crespo, N. Akhmediev, Dissipative rogue waves generated by chaotic pulse bunching in a mode-locked laser, Phys. Rev. Lett. 108 (23) (2012) 233901, https://doi.org/10.1103/PhysRevLett.108.233901.

[38] D. Korobko, R. Gumenyuk, I. Zolotovskii, O. Okhotnikov, Multisoliton complexes in fiber lasers, Opt. Fiber Technol. 20 (6) (2014) 593-609, https://doi.org/10.1016/j. yofte.2014.08.011 